\title{
Three-dimensional photonic crystals by large-area membrane stacking
}

\author{
Ling Lu,* Lin Lee Cheong, Henry I. Smith, Steven G. Johnson, John D. Joannopoulos, and Marin Soljačić \\ Research Laboratory of Electronics, Massachusetts Institute of Technology, Cambridge, Massachusetts 02139, USA \\ ${ }^{*}$ Corresponding author: linglu@mit.edu
}

Received August 13, 2012; accepted September 1, 2012;

posted October 22, 2012 (Doc. ID 174195); published November 14, 2012

\begin{abstract}
We designed and analyzed a "mesh-stack" three-dimensional photonic crystal of a $12.4 \%$ bandgap with a dielectric constant ratio of $12: 1$. The mesh-stack consists of four offset identical square-lattice air-hole patterned membranes in each vertical period that is equal to the in-plane period of the square lattice. This design is fully compatible with the membrane-stacking fabrication method, which is based on alignment and stacking of large-area single-crystal membranes containing engineered defects. A bandgap greater than $10 \%$ is preserved as long as the membranes are subjected to in-plane misalignment less than $3 \%$ of the square period. By introducing a linear defect with a nonsymmorphic symmetry into the mesh-stack, we achieved a single-mode waveguide over a wide bandwidth. (c) 2012 Optical Society of America

OCIS code: $160.5293,050.6875,130.5296,220.4241$.
\end{abstract}

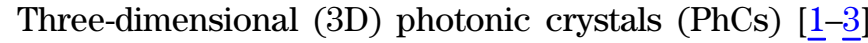
promise unprecedented control of light, but their development at optical frequencies has been limited by fabrication challenges. Recent developments $[\underline{4}, \underline{5}]$ in the membrane-stacking [ [6] technology for fabricating 3D PhCs offer the possibility of achieving centimeter-scale 3D PhCs containing controlled defects using optimal materials including single-crystal materials. Devices can be incorporated into each single-crystal membrane using planar technologies, and the membranes are then aligned and stacked to form 3D PhCs. This membrane-stacking method requires every membrane layer to be suspendible and self-supporting. In contrast, each layer of the popular woodpile structure [7] consists of disconnected rods. No previously reported $3 \mathrm{D}$ PhC design with a full bandgap $[\underline{1}, 8]$ is known to satisfy this topological constraint of suspendability without considerably complicating the fabrication process. In this Letter, we present a 3D $\mathrm{PhC}$ design ("mesh-stack") that is fully compatible with the large-area membrane-stacking fabrication approach. A single-mode waveguide design is also provided.

The mesh-stack structure is illustrated in Fig. 1(a), where five identical membranes are stacked. Each membrane in a vertical period is colored differently; the first and fifth layers (red color) are periodically equivalent. Only two materials are used: air and a high-index material of dielectric constant $(\epsilon)$ of 12 . This mesh-stack structure has the diamond lattice [8] shown in Fig. 1(b), when $c=a$. Each air hole in the mesh-stack is located at a lattice point of the diamond structure. It is well known that the diamond structure has the largest bandgap $(27.6 \%$ at $\epsilon=12)$. Likewise, the mesh-stack with the diamond structure also possesses a full 3D bandgap.

Since the layered configuration breaks the cubic symmetry along the $\hat{z}$ direction, we allow the vertical period $c$ to be away from $a$ in order to optimize the bandgap size (similar to the procedure taken for the woodpile design [7]). This changes the diamond structure from its original face-centered-cubic (fcc) lattice to the face-centered-tetragonal (fct) lattice. We define the air-hole shapes using the equation $|x|^{m}+|y|^{m}=r^{m}$, where $m$ defines the edge curvature [9] and $r$ is the "radius" of the air holes. $c, m$, and $r$ are the three free parameters. By optimizing the bandgap size for different air-hole "radii" $(r)$ [10], we found that the maxima always peak around $c=a / \sqrt{2}$, which is the lattice constant of the square lattice in each membrane. So we set the vertical period $c$ to be the in-plane square period for the rest of this Letter.

The optimized bandgap sizes and the corresponding air-hole edge curvatures $(m)$ are plotted in Fig. 1(c) for different air-hole "radii" $(r)$. The minimum vein width is $v=a / \sqrt{2}-2 r$, which is the minimum feature size of the structure. When $v$ is one-tenth of the square lattice constant, the bandgap size reaches $12.4 \%$ at $m=2.28$. This band structure is plotted in Fig. 1(d). The minimum dielectric constant needed to open up the bandgap is $\epsilon=5.5$. The fact that the optimized air-hole shapes do not contain sharp edges at the membrane-air interfaces will help relieve the stress and reduce the possibility of membrane cracking during the membrane release and stacking.

A waveguide in the mesh-stack PhC can be designed by introducing a line defect without breaking the required connectedness of the membranes. However, adding or removing dielectric material in one membrane brings several waveguide dispersion curves, close in frequencies, into the bandgap at the same time. A better design, shown in Fig. 2(a), is to extend the waveguide defects into a second membrane. We connect the air holes perpendicular to the waveguide direction, so the connectedness of the membranes is maintained. The defects are duplicated from the first layer to the next layer that is shifted along the waveguide direction (110). This introduces a glide nonsymmorphic symmetry operation that is a combination of a half lattice shift and a mirror operation in this one-dimensional periodic system. The waveguide modes are thus two-fold degenerate [11] at the edge of the Brillouin zone shown in Fig. 2(b) [12]. Importantly, the waveguide dispersion relations have nonzero slopes at the point of degeneracy, which avoids the divergent group-velocity dispersions typically arising at the edge of the Brillouin zone (due to vanishing slopes) and enables low-dispersion single-mode operation over 
(a)

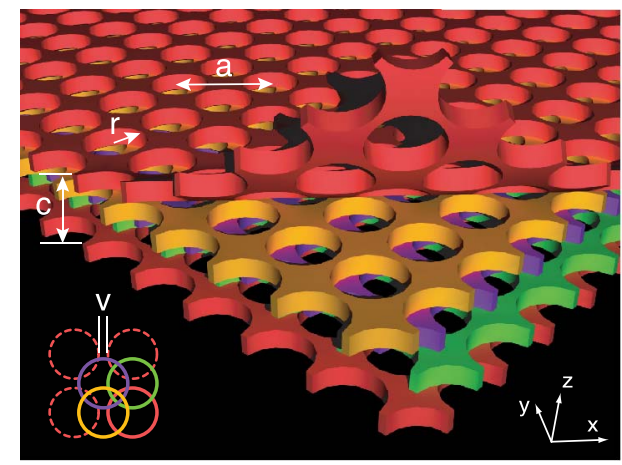

(c)

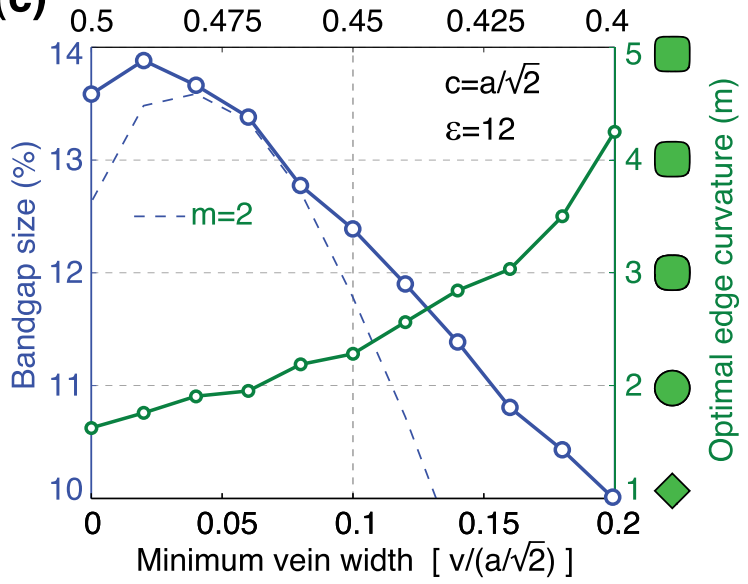

(b)
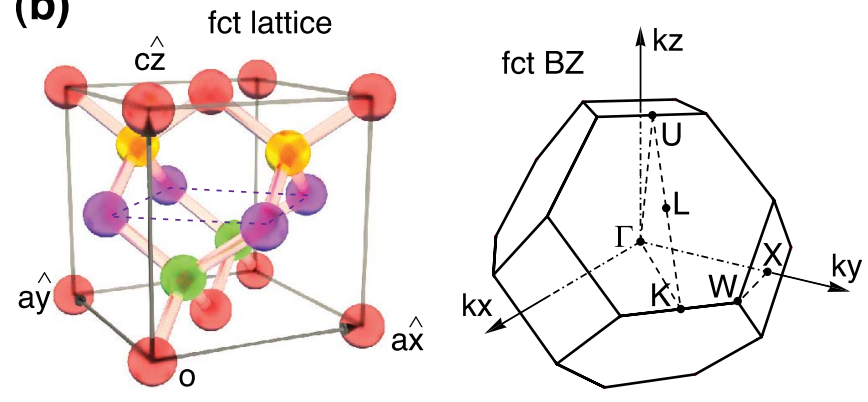

(d)

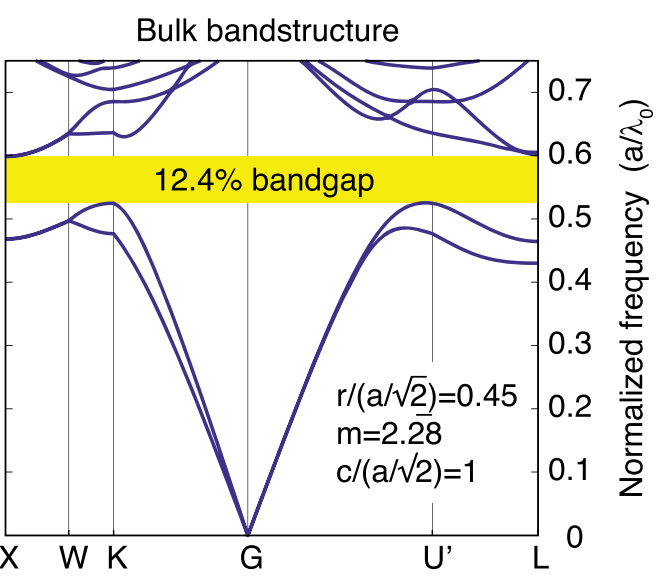

Fig. 1. (Color online) (a) To-scale illustration of the mesh-stack 3D PhC for five layers, where the first and fifth red membranes are periodically equivalent. The $r=0.45(a / \sqrt{2}), m=2$, and $c=a / \sqrt{2}$. A portion of the top red layer is peeled back to show the relative displacements of the four layers that constitute one period of the structure. The left bottom inset is a top view illustration of the inplane offsets between the four membranes. (b) Illustration of the fct lattice with a diamond-like structure. It becomes the fcc/diamond lattice when $c=a$. The colors of the spheres match the membrane colors in (a). (c) Bandgap sizes with optimized edge curvatures $(m)$ for different minimum vein widths $(\mathrm{v})$. (d) Band diagram of the optimized $\mathrm{PhC}$ at $v /(a / \sqrt{2})=0.1$.

a wide bandwidth. The distribution of the field amplitude of one of these degenerate modes is plotted in Fig. 2(c).

One possible way to further increase the bandgap of the mesh-stack is to incorporate angled etching into the fabrication. For example, instead of etching each membrane vertically, two angled etchings along the directions of the diamond bonds [in Fig. 1(b)] increases the bandgap to $18 \%$ [13]. The Yablonovite [14,15] and woodpile $[\underline{16}, \underline{17}]$ bulk $3 \mathrm{D}$ PhCs, both of $18 \%$ gap size, call for angled etching. By combining membrane stacking with angled etching, one can envision fabricating Yablonovite-like $\mathrm{PhC}$ structures in which controlled defects are introduced in any layer. Aside from the increase of bandgap size, angled etching can also make thick membranes consisting of several elementary membrane layers in the Yablonovite, woodpile, or slanted-pore [18] structures. Those thick membranes can be stacked where no defects are needed; this can potentially improve the efficiency of the fabrication.

In order for the mesh-stack [in Fig. 1(d)] to have a central bandgap wavelength of $1.55 \mu \mathrm{m}$, both the vertical period and the in-plane period of the square lattice $(a / \sqrt{2})$ need to be $616 \mathrm{~nm}$. The thickness of each membrane is $154 \mathrm{~nm}(0.25 a / \sqrt{2})$. The smallest dimension for lithography is $61.6 \mathrm{~nm}(v=0.1 a / \sqrt{2})$, which is the width of the dielectric vein between two neighboring air holes. The offset distance between successive membranes is $308 \mathrm{~nm}(0.5 a / \sqrt{2})$. To study the effects of membrane misalignment, we applied random in-plane offsets to 12 membrane layers in the super-cell calculations [19]. The standard deviation of the Gaussian random offsets is $\sigma$ along both in-plane axes. Ten calculations were done for each $\sigma$; the mean values and standard deviations of the bandgap sizes and central frequencies are plotted in Fig. 3. In order to have a bandgap size above $10 \%$, alignment accuracy better than $3 \%$ of the in-plane lattice constant $a / \sqrt{2}$ is required; this corresponds to a maximum alignment error of $18.5 \mathrm{~nm}$ at $1.55 \mu \mathrm{m}$. Fortunately, less than $1 \mathrm{~nm}$ detectivity has been demonstrated by an interferometry-based alignment technique [20].

We believe the mesh-stack design can help reduce the challenges of fabricating 3D PhCs, especially in the membrane-stacking approach.

We thank Shabnam Ghadarghadr, Corey P. Fucetola, Euclid E. Moon, Wenjun Qiu, and Song Liang Chua for helpful discussions. L.L.C and H.I.S were supported by the Air Force Office of Scientific Research under Grant No. FA9550-08-1-0379. This work was supported in part 
(a)
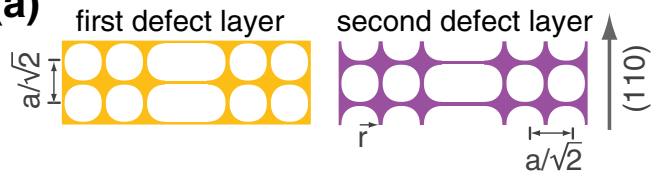

(b)

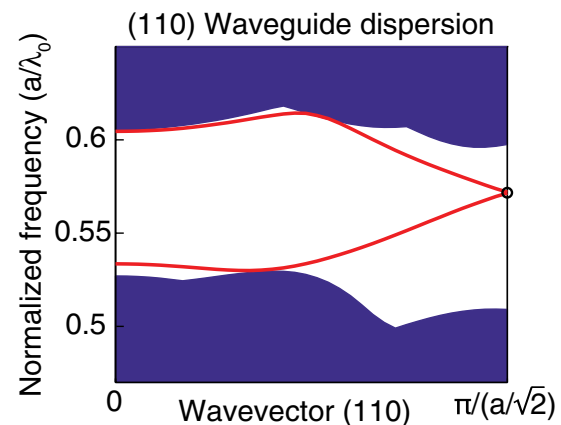

(c)

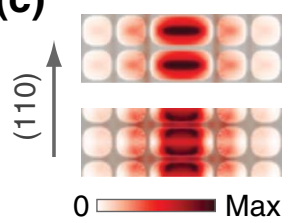

$|\mathrm{E}|$

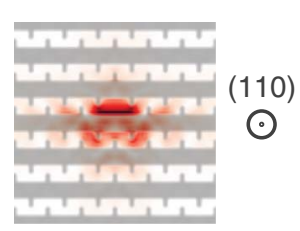

Fig. 2. (Color online) (a) Two consecutive membranes containing identical defects as waveguide cores. (b) Dispersion relations of the nonsymmorphic waveguide. (c) Field profiles of one of the degenerate modes at $k=\pi /(a / \sqrt{2})$ plotted in a linear scale overlaid on the dielectric distribution in transparent gray color.

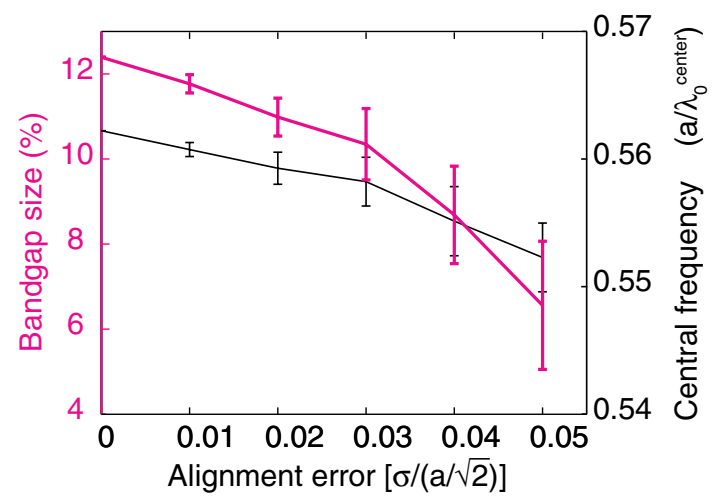

Fig. 3. (Color online) Mesh-stack bandgap sizes and central frequencies as a function of the alignment offsets of the membranes. $\sigma$ is the standard deviation of the random offsets along both in-plane directions. by the U.S.A.R.O. through the ISN, under Contract No. W911NF-07-D-0004. L.L. was supported in part by the MRSEC Program of the NSF under Award No. DMR-0819762. L.L. and M.S. were partially supported by the MIT S3TEC Energy Research Frontier Center of the Department of Energy under Grant No. DESC0001299.

\section{References and Notes}

1. J. D. Joannopoulos, S. G. Johnson, J. N. Winn, and R. D. Meade, Photonic Crystals: Molding the Flow of Light, 2nd ed. (Princeton University, 2008).

2. E. Yablonovitch, Phys. Rev. Lett. 58, 2059 (1987).

3. S. John, Phys. Rev. Lett. 58, 2486 (1987).

4. S. Ghadarghadr, C. P. Fucetola, L. L. Cheong, E. E. Moon, and H. I. Smith, J. Vac. Sci. Technol. B 29, 06F401 (2011).

5. A. A. Patel and H. I. Smith, J. Vac. Sci. Technol. B 25, 2662 (2007).

6. K. Aoki, H. T. Miyazaki, H. Hirayama, K. Inoshita, T. Baba, K. Sakoda, N. Shinya, and Y. Aoyagi, Nat. Mater. 2, 117 (2003).

7. K. Ho, C. Chan, C. Soukoulis, R. Biswas, and M. Sigalas, Solid State Commun. 89, 413 (1994).

8. M. Maldovan and E. L. Thomas, Nat. Mater. 3, 593 (2004).

9. $m$ is an arbitrary real number. When $m=2$, the equation defines a circle. When $m=1$, it defines a diamond. When $m=\infty$, it defines a square. This is illustrated on the right side of Fig. 1(c).

10. The bandstructure calculations were done using MIT Photonic-Bands package (MPB, http://ab-initio.mit.edu/ $\mathrm{mpb}$ ) and the optimizations were done using the nonlinear optimization package (NLopt, http://ab-initio.mit.edu/nlopt).

11. A. Mock, L. Lu, and J. O’Brien, Phys. Rev. B 81, 155115 (2010).

12. This degeneracy, protected by the glide spatial symmetry, ensures the continuity of the bands across the Brillouin zone boundary, while the continuities of the dispersion curves inside the Brillouin zone are protected by translational spatial symmetry.

13. K. Aoki, Appl. Phys. Lett. 95, 191910 (2009).

14. E. Yablonovitch, T. J. Gmitter, and K. M. Leung, Phys. Rev. Lett. 67, 2295 (1991).

15. C. C. Cheng and A. Scherer, J. Vac. Sci. Technol. B 13, 2696 (1995).

16. S. Takahashi, K. Suzuki, M. Okano, M. Imada, T. Nakamori, Y. Ota, K. Ishizaki, and S. Noda, Nat. Mater. 8, 721 (2009).

17. L. Tang and T. Yoshie, J. Vac. Sci. Technol. B 28, 301 (2010).

18. O. Toader and S. John, Phys. Rev. E 71, 036605 (2005).

19. S. G. Johnson and J. D. Joannopoulos, Opt. Express 8, 173 (2001).

20. E. E. Moon, M. K. Mondol, P. N. Everett, and H. I. Smith, J. Vac. Sci. Technol. B 23, 2607 (2005). 